\title{
Study on the Clinical Characteristics of Hand-Foot-Mouth Disease Caused by Enterovirus 71 and Other Enteroviruses
}

\author{
Yuan Ren \\ Baoshan College of Traditional Chinese Medicine, Baoshan, Yunnan, 678000
}

Keywords: clinical characteristics; hand-foot-mouth disease; enterovirus 71

\begin{abstract}
To investigate the clinical features of hand-foot-mouth disease caused by EV71 in children with hand-foot-mouth disease and other enteroviruses, the clinical data of pediatric hand-foot-and-mouth disease (HFMD) were retrospectively analyzed in all hospitals designated for etiology. Screening for EV71-infected hand-foot-mouth disease was analyzed, with other hand-foot-mouth disease patients caused by enterovirus as a control group.
\end{abstract}

\section{Introduction}

To investigate the clinical features of hand-foot-and-mouth disease caused by EV71 in children with hand-foot-mouth disease and other enteroviruses. METHODS: The clinical data of pediatric hand, foot and mouth disease (HFMD) were collected retrospectively. The EV71-infected hand-foot-mouth disease children were screened for analysis. Other hand-foot-mouth disease patients caused by enteroviruses as a control group.

Enterovirus 71 (EV71) is one of the major pathogens of childhood hand-foot-mouth disease (HFMD) and central nervous system infections [1]. Studies have shown that, compared with other virus serotypes such as Coxsackie A16 (COXA16), EV71 infection is more likely to cause central nervous system damage, acute pulmonary edema, etc. [2], morbidity and mortality are higher. In addition, it also attacks the skin, mucous membranes, and myocardium. It does not involve the kidneys and the liver basically. Among the cases related to severe hand-foot-and-mouth disease in the past years, EV71 is crucial. In order to detect severe cases early, improve cure rate, and reduce the incidence of sequelae, we retrospectively analyzed clinical data of 320 hand, foot and mouth disease children who were positive for hospitalization etiology tests in all designated hospitals in Heze district in March 2009 and September 2009. This included 106 children with EV71 infection and 214 children with other enterovirus infections. Compare the differences in clinical symptoms, signs, and laboratory tests, and explore possible factors that influence the prognosis, and look for indicators that can predict early onset of severe disease.

\section{Materials and Methods}

According to the 2008 "Hand-Foot-Mouth Disease Prevention and Control Guideline", the diagnostic criteria for hand-foot-and-mouth disease (HFMD) and 37 etiologically positive patients were used as the research object. According to the results of etiological screening, there were 28 cases of EV71 infection group and 9 cases of other enterovirus infection groups. The EV71 infection group included 17 males and 11 females. The age ranged from 8 to 49 months. The median age was 30 months, and 75\% were less than 3 years old. Other enterovirus infections included 8 males and 1 female, aged 15 to 40 months. The median age was 34 months. $89 \%$ of the patients were younger than 3 years of age. Among them, 5 were detected with Coxsackie A16 virus. Enterovirus in 4 cases.

The clinical data of 2 groups of patients were collected to analyze and compare the fever, rash, main laboratory test indexes (peripheral blood leukocyte count, blood glucose, CK-MB, lactate dehydrogenase and CD4+/CD8+ ratio) and concurrence in the 2 groups. The condition of the disease. Use SPSS13.0 statistical software for data processing. Normally distributed measurement data are expressed as $(\mathrm{x} \pm \mathrm{s})$ and t-tests are used. Measured data with non-normal distribution are 
expressed as full-distance (median) and rank sum tests are used. Count data were compared using Fisher's exact probability method.

\section{Results and Discussion}

Both groups had fever. The EV71 infection group had a fever of $(5.1 \pm 1.7)$ days and a peak body temperature of $(39.1 \pm 0.7){ }^{\circ} \mathrm{C}$. Other enterovirus infections were (3.2 \pm 1.0$)$ days and (38.5 \pm 0.7$)$ respectively. $)^{\circ} \mathrm{C}$, there was a statistically significant difference in the peak and body temperature peaks between the two groups (all $\mathrm{P}<0.05$ ).

In both groups, there were rashes. In the EV71 infection group, 5 cases (18\%) were given rash at the head of the mouth, and 23 cases (82\%) were hereditary rash areas with hands and feet, and 6 cases were herpes hernia (21\%).), with 22 cases (79\%) of minor millet rash; 6 cases (6/9), 3 cases (3/9), 6 cases (6/9), and 3 cases of other enterovirus infections (3/9), the ratio of the first oral rash in the two groups and the predominant proportion of herpes were significantly different $(\mathrm{P}<0.05)$. Respiratory complications occurred in 19 cases (68\%) of the V71 infection group, including 8 cases of pneumonia, 11 cases of acute tracheal-bronchitis, 5 cases of central nervous system complications (18\%), and 2 cases of viral meningitis. There were 2 cases of viral encephalitis and 1 case of meningoencephalitis. Respiratory complications occurred in 4 patients (4 of 9) in other enterovirus infections, 4 in acute tracheobronchitis, and 1 in patients with central nervous system damage (1 of 9). Viral encephalitis was diagnosed (pathogens The results are unclassified enteroviruses). There was no significant difference in the incidence of respiratory complications and central nervous system damage between the two groups $(\mathrm{P}>0.05)$.

In 2008, the pathogen detected in the hand-foot-mouth disease epidemic in Beijing was dominated by EV71. By comparing the differences in clinical manifestations between the EV71-infected group and other enterovirus-infected groups, we found that the EV71-infected group had a significantly longer heat duration than other enterovirus-infected groups, and that the EV71-infected group had a higher peak body temperature and peaked at an average value. $39.1{ }^{\circ} \mathrm{C}$, suggesting that children with EV71 infection is more serious than other enterovirus infections. As for the rash sequence, the EV71 infection group was more likely to have the rash area headed by the hands and feet, while other enterovirus infection groups were often given the oral area and the rash area. In addition, in this study, the EV71 infection group had a smaller rash, the rash type was dominated by minor millet rashes, and the other enterovirus infection groups were mainly herpes. This is consistent with the literature [3]. Enteroviruses mainly invade the skin, mucous membranes, central nervous system, lungs, and myocardium, and rarely affect the kidneys and liver [4]. Therefore, we only compared the peripheral blood leukocyte count, blood glucose, CK-MB, lactate dehydrogenase, and CD4+/CD8+ ratio to 5 indicators in 2 groups. The results showed that there was no significant difference in the above-mentioned laboratory tests between the two groups $(\mathrm{P}>0.05)$. Acute respiratory infection is a common complication of EV71 infection. It has been reported in Australia, Canada, and the EV71 epidemic in Taiwan, China in 1998. It includes pharyngitis, acute tracheobronchitis, and pneumonia. Most cases require hospitalization [5]. In this study, the incidence of respiratory complications in the EV71-infected group was similar to that of other enterovirus infections.

Hand-foot-mouth disease can develop in all four seasons, but the main onset season is summer and autumn. The virus spreads through respiratory and digestive tracts through hand-foot-mouth disease patients and virus carriers. So far, there are more than 20 viruses that can cause virus-induced hand-foot-mouth disease (HFMD). However, two common types of enteroviruses, CoxA16 and EV71, are predominant in clinical infections. The viral load of hand-foot-mouth disease caused by two different types of enterovirus infections is different from the clinical features, and the treatment methods are also different. Accurate understanding of the clinical features of hand-foot-mouth disease caused by CoxA16 and EV71 viruses is the basis for effective treatment of hand-foot-and-mouth disease. The treatment of hand, foot and mouth disease requires analysis of the virus that it infects, but viral RNA detection takes a long time, which in some cases limits the timely treatment of children with hand, foot and mouth disease. Therefore, clinical experience is 
particularly important for the early treatment of hand, foot and mouth disease. The clinical experience of medical staff mainly comes from the clinical characteristics of children with hand, foot and mouth disease caused by different enterovirus infections. The results of this study suggest that the EV71 virus concentration is significantly higher than the CoxA16 virus concentration, and the virus load is closely related to the infected virus. This is similar to the results of many studies in China. Moreover, studies have pointed out that there is no obvious link between the viral load and the patient's condition. Therefore, it is necessary to promptly give appropriate antiviral drugs for hand, foot and mouth disease caused by different viruses, and increase or decrease the dose of antiviral drugs according to the type of virus so as to avoid insufficient doses or improper use of drugs to produce antiviral drugs. Treating hand, foot and mouth disease brings new challenges. The duration and duration of heat in the EV71 group were longer than those in the CoxA16 group. The proportion of cough in the EV71 group was significantly lower than that in the CoxA16 group. The proportion of children in the EV71 group with somnolence, convulsions, vomiting, and muscle spasm was significantly higher than that in the CoxA16 group. Statistical significance $(\mathrm{P}<0.05)$. The results of the study suggest that the disease in children infected with EV71 is more severe than that in children with CoxA16, and the incidence is less noticeable. As a result, both the duration of illness and the heat increase significantly [6]. CoxA16 disease is more easily noticeable, timely detection and timely diagnosis and treatment, this may also be an important reason for the low viral load of CoxA16. Moreover, children with EV71 infection had lower cough, more drowsiness, increased convulsions, increased vomiting, and increased muscle mass. In the clinical treatment of early hand, foot and mouth disease these obvious clinical features can provide clear guidance for the initial judgment of physicians, hand, foot and mouth disease, the incidence of rapid, if not treated in time may be life-threatening, timely clinical treatment of hand, foot and mouth Provide valuable time. The results suggest that the vital signs of children with HFMD induced by the EV71 virus are worse than those in the CoxA16 group. The possible reason is that the children in the EV71 group are difficult to be detected at the early stage of the disease, and the children have limited expression capacity. Parents failed to detect the condition in time. The incidence of hand, foot and mouth disease is rapidly progressing, and the clinical manifestations are diverse. When the parents of children with the disease have found that the disease has progressed to the middle or even late stage, the children's vital signs are all poor. Therefore, more attention should be paid to the clinical treatment of children with EV71. The incidence of oral ulcers in the upper eyelid, pharyngeal isthmus, and buccal mucosa of the EV71 group was significantly lower than that of the CoxA16 group, and the difference was statistically significant. The results suggest that the incidence of oral ulcers in the CoxA16 group was significantly higher than that in the EV71 group. The possible reason was that the CoxA16 virus infection mainly damaged the skin and mucous membranes. For children with more oral ulcers and concomitant coughing, it may be related to CoxA16 infection.

\section{Conclusion}

Compared with other intestinal enterovirus HFMD, hand-foot-mouth disease caused by EV71 infection is severe, and it is more likely to combine central lesions and pulmonary edema. If it is not timely to find treatment, the disability rate and mortality are higher. Therefore, the analysis and summary of the clinical data of pediatric HFMD in Heze area will help us to discover the severe cases of EV71 as early as possible during the epidemic of HFMD in order to reduce the morbidity and mortality of the disease.

\section{References}

[1] Schmidt NJ, Lennette EH, Ho HH. An apparently new enterovirusisolated from patients with disease of the central nervoussystem [J], JInfect Dis, 1974, 129: 304-309

[2] Chang LY, Huang LM, Gau SS, et al. Neurodevelopment and cognition in children after enterovirus 71 infection [J]. N Engl J Med, 2007, 356:1226-1234 
[3] Kow TC, HsiaoLC, Shan TW, et a1. Epidemiologic features of hand-foot-mouth disease and herpangina caused by enterovirus 71 inTaiwan. 1998-2005 [J]. Clin Virol, 2006, 37:47-52

[4] Yang Shaoji. Enterovirus 71 infection [J]. New Medicine, 2008,39 (6):354-355,366 Yang Shao-ji. Enterovirus 71 Infection [J]. New Medicine, 2008,39 (6): 354-355,366 (In Chinese)

[5] Tang Hongping. Clinical analysis of 42 cases of severe hand-foot-mouth disease[J]. New Medicine, 2008,39 (11):718TANG Hong-ping. Clinical Analysis of 42 cases with hand-foot-mouth disease[J]. New Medicine, 2008, 39 (11): 718 (In Chinese)

[6] Lin H, Hwang KP, Ke GM, et al. Evolution of EV-71 genogroup in Taiwan from 1998 to 2005; an emerging of sub genogroup C4 ofEV-71 [J], J med Virol, 2006, 78::254 -262 